\title{
Dual Ureaplasma parvum arthritis: a case report of $U$. parvum septic arthritis following contralateral reactive arthritis in an immunosuppressed patient
}

Lea Lemoine ${ }^{2,5^{*}}$ (D) Cecile Le Brun ${ }^{3}$, Francois Maillot ${ }^{2,4,5}$, Camille Thorey ${ }^{1}$, Annaelle Boucaud ${ }^{2,5}$, Adrien Lemaignen ${ }^{1,5}$ and Adrien Bigot ${ }^{2}$

\begin{abstract}
Background: Ureaplasma parvum is usually part of the normal genital flora. Rarely can it cause invasive infections such as genitourinary infections, septic arthritis, or meningitis.

Case presentation: Here we present the first description of chronic ureterocystitis in a 56-year-old immunocompromised patient, complicated first by reactive arthritis and secondarily by contralateral septic arthritis due to U. parvum infection. U. parvum was detected in synovial fluid and in a urine sample. Treatment consisted of double-J stenting and targeted antibiotic therapy. Evolution showed resolution of urinary symptoms and clinical improvement of arthritis despite functional sequelae.

Conclusions: Given the high prevalence of U. parvum colonisation, this diagnosis should remain a diagnosis of exclusion. However, because of the difficulty in detecting this microorganism, it should be considered in unexplained subacute urethritis or arthritis, including reactive arthritis, especially in immunosuppressed patients. Real-time PCR positivity in the absence of a differential diagnosis should not be overlooked.
\end{abstract}

Keywords: Ureaplasma parvum, Reactive arthritis, Septic arthritis, Immunosuppression, Case report

\section{Background}

In 1954, Shepard et al., discovered that species from the genera Ureaplasma, part of the Mycoplasmataceae family together with Mycoplasma, were pathogens causing non-gonococcal urethritis [1]. Ureaplasma urealyticum and Ureaplasma parvum are the main species identified as pathogenic and were identified in 1999 [2] .

$U$. urealyticum is a common pathogen in genitourinary infections, reactive arthritis [3], and occasionally septic arthritis [4]. In contrast, $U$. parvum is usually

\footnotetext{
${ }^{*}$ Correspondence: lea.lemoine@live.fr
}

${ }^{5}$ Faculty of Medicine of Tours, François Rabelais University, 10 Boulevard

Tonnellé, Tours, France

Full list of author information is available at the end of the article considered part of the normal genital flora and therefore non-pathogenic. Nevertheless, it can occasionally be an opportunistic or iatrogenic [5] pathogen and be responsible for genital tract infections [6] or respiratory disease in newborns [7]. Given the prevalence of colonisation in the female genital tract, which is up to $40 \%[8]$, U. parvum positive screening on multiplex real-time PCR for sexually transmitted infection (STI) is usually not reported because of lack of clinical significance. However, a few reports of $U$. parvum infections, including post-surgical meningitis [4] and septic arthritis but not reactive arthritis, have been described to date. 
Here, we report a case of $U$. parvum septic arthritis following reactive arthritis as a complication of genitourinary infection in an immunosuppressed woman.

\section{Case presentation (see Additional file 1: Timeline)}

A 56-year-old woman was admitted in March 2019 for diagnostic work up. She had received treatment for follicular lymphoma with R-CHOP from October 2017 to April 2018 and was still on rituximab. She had no other significant medical history.

She first presented in April 2018 with arthritis of the right knee and received ceftriaxone, then cefpodoximeproxetil as a probabilistic antibiotic therapy, with favourable outcome. Arthrocentesis was not performed.

In October 2018, the patient reported dysuria and haematuria. Urine tests showed leukocyte count $>1000 / \mathrm{mm}^{3}$ and red cell count $>1000 / \mathrm{mm}^{3}$, but culture remained sterile. Bladder ultrasonography revealed vesical thickening and intravesical clot deposits, evocative of haemorrhagic cystitis. The patient declined cystoscopy and received empirical treatment with furadantine $50 \mathrm{mg}$ target dose for 5 days.

In November, she presented left-knee arthritis, recurrence of haemorrhagic cystitis and bilateral conjunctivitis. The right knee was clinically normal. Arthrocentesis showed an inflammatory liquid (leukocyte count $12,600 / \mathrm{mm}^{3}$ ) with no crystals and negative Gram stain. Cultures remained sterile; broad-range $16 \mathrm{~S}$ ribosomal RNA PCR of synovial fluid was negative. Reactive arthritis was considered, but urine samples were negative for Chlamydia trachomatis, Neisseria gonorrhoeae and Mycoplasma genitalium. STI multiplex real-time PCR of a urine sample was positive for $U$. parvum on retrospective analysis (not done at that time). No antibiotics were administered, and the evolution was favourable without sequelae.

In March 2019, the cystitis worsened, and fever recurred. The patient was admitted with contralateral (right) knee arthritis and acute renal failure. The left-knee examination was unremarkable at that time. CT revealed cystitis and bilateral ureteral stenosis responsible for dilatation of the urinary tract (Fig. 1b). CT did not reveal any stone formation.

Double-J stents were placed and ceftriaxone was initiated. Blood and urine culture remained sterile and fever persisted. Right knee puncture retrieved purulent liquid, with leukocyte count $1620000 / \mathrm{mm}^{3}$ (80\% neutrophils, negative Gram stain). Then, hypotension developed: piperacilline-tazobactam was started, surgical knee drainage was performed, and intracellular arthritis was suspected. Multiplex STI real-time PCR (Allplex STI Essentials, Seegene), was strongly positive for $U$. parvum on synovial fluid and on a urine sample. Specific culture media was inoculated (A7agar, Biomérieux and MYCOFAST RevolutioN ElitechGroup), and A7 agar culture was positive for $U$. parvum in urine sample, but antibiotic susceptibility testing was negative. Bladder biopsy showed aspecific inflammation; PCR was negative for $U$. parvum although after 5 days of doxycycline treatment. 18 F-fluoro-2-deoxy-D-glucose positron emission tomography showed right-knee and right latero-uterine hypermetabolism (Fig. 1a), but the left knee

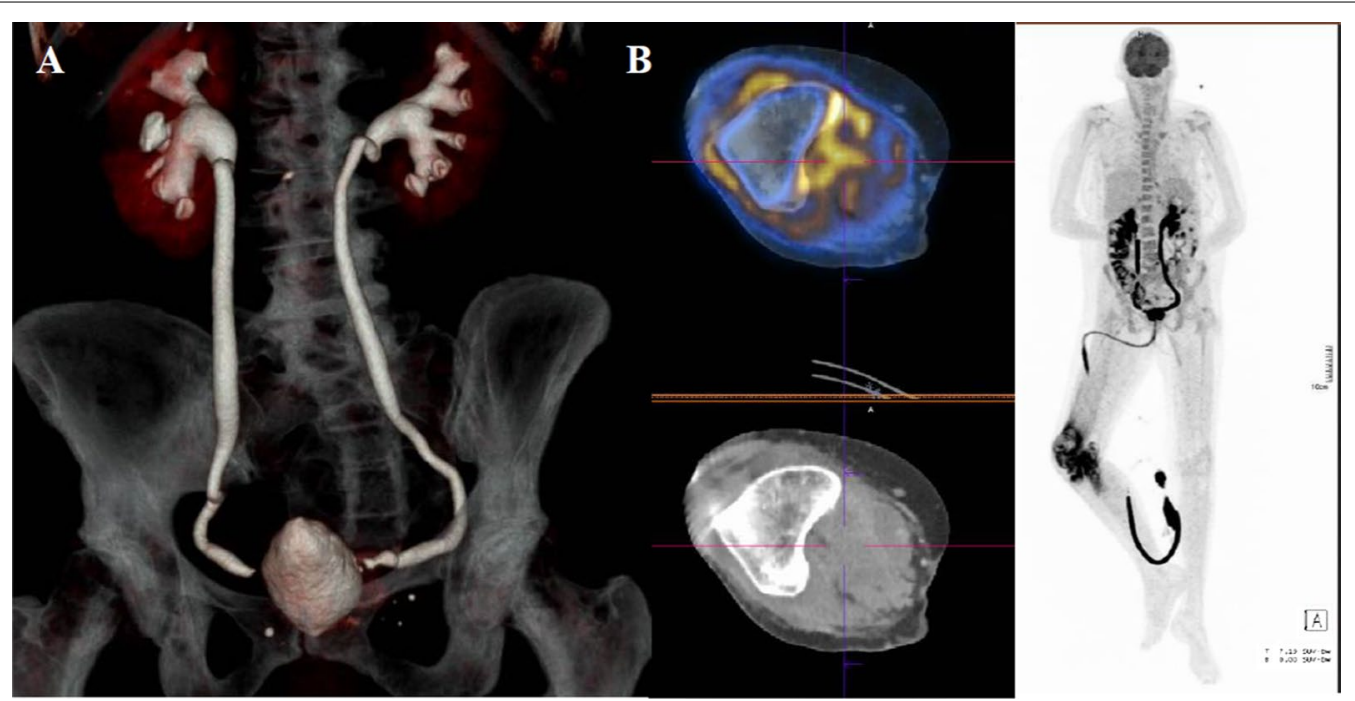

Fig. 1 a Positron emission tomography tomodensitometry showing hypermetabolism of the right knee (maximal standardized uptake value [SUVmax]: 11.8) and right latero-urine hypertabolism (SUVmax: 7.5). b Computed tomography showing dilatation of urinary tract and bilateral ureteral stenosis 
was not hypermetabolic. Uterine echoendoscopy was non-contributive.

The diagnosis retained was $U$. parvum subacute ureterocystitis complicated by oculo-urethro-synovial syndrome (reactive arthritis) and subsequently by obstructive renal failure and septic arthritis. Antibiotic therapy with doxycycline $100 \mathrm{mg}$ twice a day was started for 3 months. The fever receded, but local inflammation persisted. A control arthrocentesis after 15 days without antibiotics showed persistent arthritis but negative PCR results for $U$. parvum. Clarithromycin was introduced for 6 weeks, with marked clinical improvement: signs of erythema, swelling and pain disappeared, despite functional sequelae (flexion limitation $90^{\circ}$ ). Antibiotic treatment was well tolerated, and no clinical or biological event appeared during treatment.

\section{Discussion and conclusions}

We report a case of right-knee reactive arthritis that was spontaneously regressive without sequelae. The rightknee arthritis preceded left-knee septic osteoarthritis associated with bilateral ureteral stenosis secondary to chronic ureterocystitis due to $U$. parvum infection.

$U$. parvum colonisation of the urogenital tract has been reported, and a high load of $U$. parvum has been found associated with chronic non-gonococcal urethritis in men, although whether it was a cause or a marker of non-gonococcal urethritis was unclear [9]. Currently, the paucity of documented $U$. parvum infection suggests opportunistic pathogenicity. Indeed, most infections have been reported in immunosuppressed or post-surgical patients. Ureaplasma are a common observation in patient with B-cell specific dysfunction such as patients with Common Variable Immune Deficiency [10]. For example, the first $U$. parvum prosthetic joint infection [11] was reported in 2014 in a patient withhypogammaglobulinemia, obesity and diabetes. Native joint infections have often been reported as part of systemic infections: bilateral knee arthritis with aortic co-infection by $U$. parvum and Mycoplasma hominis [12] as well as fatal mycotic aneurysms was reported in a 54-year-old man with lymphoma. Korytny et al. reported $U$. parvum orchitis complicated by gleno-humeral arthritis and endocarditis in a 56-year-old man with lymphoma history [13]. However, isolated chronic genitourinary infection by $U$. parvum has never been reported.

In the present case, retrospective assessment of the November 2018 STI multiplex real-time PCR of a urine sample was positive for $U$. parvum. However, given the frequency of genital colonization [8] and the usual absence of pathogenicity of $U$. parvum, this result was not considered significant, and only C. trachomatis, $N$. gonorrhoeae and M. genitalium results were reported. In the absence of other causal pathogens, clinical presentation and evolution pointed towards a diagnosis of $U$. parvum cystoureteritis at that time. Hence, we suggest that testing for $U$. parvum in subacute cystitis/ureteritis should be considered in immunosuppressed patients after excluding usual (including intracellular) pathogens and mycobacterial sampling.

Ureasplama sp. are not observed on Gram staining, and cultures are only contributory for high bacterial load and require specific media. Moreover, classical identification techniques may require 3 to 7 days, whereas realtime PCR is faster and more sensitive for Ureaplasma detection [14]. Use of next generation sequencing (NGS) is also a valuable technique to detect microbial cell-free DNA [15] and sometimes even if antibiotic was used [5]. Therefore, PCR should be combined with specific media culture and NGS should be considered. As ureaplasma is deprived of cell walls, antibiotics of the beta-lactam group are not effective. Conversely, doxycycline and clarithromycin are highly effective antibiotic, with low rates of resistance reported. About $7.5 \%$ U. parvum are resistant to doxycycline by carrying the resistance gene tet(M) [16].

In our case, overlooking $U$. parvum as a possible etiology for the reactive arthritis delayed the diagnosis and allowed septic complications to develop. Indeed, in November, the patient presented left-knee arthritis associated with cystitis and conjunctivitis (oculo-urethrosynovial syndrome). Direct examination, culture and $16 \mathrm{~S}$ real-time PCR of arthrocentesis fluid gave negative results. This episode receded in the absence of antibiotic treatment. Together with spontaneous healing, the association of conjunctivitis and ureterocystitis with a sterile arthritis suggested reactive arthritis to $U$. parvum ureterocystitis.

Reactive arthritis is a rare immune-mediated arthritis following a genitourinary or gastrointestinal infection in predisposed patients and corresponds to the classic clinical triad: urethritis, eye disorders and arthritis. The principal microorganism involved in genitourinary infections responsible for reactive arthritis is $C$. trachomatis, but Salmonella spp., Shigella spp., Campylobacter jejuni and Yersinia spp. are also frequently involved. Chlamydophila pneumoniae, Clostridium difficile, HIV and U. urealyticum [4] have been described as uncommon inciting agents of reactive arthritis.

To our knowledge, we report the first case of oculourethro-synovial syndrome due to $U$. parvum ureterocystitis in an immunosuppressed patient.

In immunosuppressed people, arthritis can be caused by mycoplasma species. Cultures of $U$. parvum are not relevant, and PCR analysis is the best way to diagnosis the infection. PCR analysis of synovial liquid should be 
performed for immunosuppressed patients, especially in the presence of gynaecologic or urinary symptoms. $U$. parvum is also likely responsible for reactive arthritis.

It did not need formal ethics approval as this complies with national guidelines ("Loi n ${ }^{\circ} 78-17$ du 6 janvier 1978 relative à l'informatique, aux fichiers et aux liberté and " Règlement (UE) 2016/679 du Parlement européen et du Conseil du 27 avril 2016, relatif à la protection des personnes physiques à l'égard du traitement des données à caractère personnel et à la libre circulation de ces données ») .

\section{Abbreviations}

STI: Sexually transmitted disease; R-CHOP: Rituximab, cyclophosphamide, doxorubicin, vincristine, and prednisone.

\section{Supplementary Information}

The online version contains supplementary material available at https://doi. org/10.1186/s12879-021-06733-0.

Additional file 1. Timeline.

\section{Acknowledgements}

We thank Laura Smales for editing comments on the manuscript.

\section{Authors contributions}

$\mathrm{LL}$ and $\mathrm{AB} 2$ were responsible for the patient's care during the diagnosis process and were major contributors in writing the manuscript. CLB performed microbiology analyses and wrote the microbiology part of the manuscript. CT helped collected information about the case as the physician who followed the patient. AL and AA1 contributed to the conception of the work and collected data on patients with U. parvum arthritis. FM revised the manuscript. All authors read and approved the final manuscript

\section{Funding}

No funding.

\section{Availability of data and materials}

Materials analysed during the current study are not publicly available because of patient privacy concerns but are available from the corresponding author on reasonable request.

\section{Declarations}

Ethics approval and consent to participate Not applicable.

\section{Consent to publication}

Written consent was obtained from the patient to publish her clinical details and images. a copy of the consent document is available for the editor of this journal

\section{Competing interests}

The authors declare that they have no competing interests.

\section{Author details}

${ }^{1}$ Department of Infectiology, University Hospital of Tours, 2 Boulevard Tonnellé, Tonnellé, France. ${ }^{2}$ Department of Internal Medicine and Clinical Immunology, University Hospital of Tours, 2 Boulevard Tonnellé, Tonnellé, France. ${ }^{3}$ Department of Bacteriology-Virology-Hygiene, University Hospital of Tours,
2 Boulevard, Tonnellé, France. ${ }^{4}$ UMR INSERM 1253, 10 Boulevard Tonnellé, Tours, France. ${ }^{5}$ Faculty of Medicine of Tours, François Rabelais University, 10 Boulevard Tonnellé, Tours, France.

Received: 8 March 2021 Accepted: 24 September 2021

Published online: 29 October 2021

\section{References}

1. Waites KB, Katz B, Schelonka RL. Mycoplasmas and Ureaplasmas as Neonatal Pathogens. Clin Microbiol Rev. 2005;18(4):757-89.

2. kong F, James G, Ma Z, Gordon S, Bin W, Gilbert GL. Phylogenetic analysis of Ureaplasma urealyticum-support for the establishment of a new species, Ureaplasma parvum. Int J Syst Bacteriol. 1999;49:1879-89.

3. Stavropoulos PG, Soura E, Kanelleas A, Katsambas A, Antoniou C. Reactive arthritis. J Eur Acad Dermatol Venereol JEADV. 2015;29(3):415-24.

4. George MD, Cardenas AM, Birnbaum BK, Gluckman SJ. Ureaplasma septic arthritis in an immunosuppressed patient with juvenile idiopathic arthritis. J Clin Rheumatol Pract Rep Rheum Musculoskelet Dis juin. 2015;21(4):221-4

5. Pailhoriès $\mathrm{H}$, Chenouard R, Eveillard M, Kempf M, Pereyre S, Bébéar C, et al A case of Ureaplasma parvum meningitis in an adult after transphenoidal ablation of craniopharyngioma. Int J Infect Dis IJID Off Publ Int Soc Infect Dis. 2019;84:5-7.

6. Combaz-Söhnchen N, Kuhn A. A Systematic Review of Mycoplasma and Ureaplasma in Urogynaecology. Geburtshilfe Frauenheilkd. 2017:77(12):1299-303.

7. Cassell GH, Waites KB, Watson HL, Crouse DT, Harasawa R. Ureaplasma urealyticum intrauterine infection: role in prematurity and disease in newborns. Clin Microbiol Rev. 1993:6(1):69-87.

8. Leli C, Mencacci A, Latino MA, Clerici P, Rassu M, Perito S, et al. Prevalence of cervical colonization by Ureaplasma parvum, Ureaplasma urealyticum, Mycoplasma hominis and Mycoplasma genitalium in childbearing age women by a commercially available multiplex real-time PCR: An Italian observational multicentre study. J Microbiol Immunol Infect Wei Mian Yu Gan Ran Za Zhi. 2018:51(2):220-5.

9. Frølund $M$, Lidbrink $P$, Wikström A, Cowan S, Ahrens P, Jensen JS. Urethritis-associated Pathogens in Urine from Men with Non-gonococcal Urethritis: A Case-control Study. Acta Derm Venereol. 2016;96(5):689-94.

10. Bloom KA, Chung D, Cunningham-Rundles C. Osteoarticular infectious complications in patients with primary immunodeficiencies. Curr Opin Rheumatol. 2008;20(4):480-5.

11. Farrell JJ, Larson JA, Akeson JW, Lowery KS, Rounds MA, Sampath R, et al. Ureaplasma parvum prosthetic joint infection detected by PCR. J Clin Microbiol. 2014:52(6):2248-50.

12. MacKenzie CR, Nischik N, Kram R, Krauspe R, Jäger M, Henrich B. Fatal outcome of a disseminated dual infection with drug-resistant Mycoplasma hominis and Ureaplasma parvum originating from a septic arthritis in an immunocompromised patient. Int J Infect Dis. 2010;14:e307-9.

13. Korytny A, Nasser R, Geffen Y, Friedman T, Paul M, Ghanem-Zoubi N. Ureaplasma parvum causing life-threatening disease in a susceptible patient. BMJ Case Rep. 2017 [cité 7 mars 2021];2017. Disponible sur: https://www. ncbi.nlm.nih.gov/pmc/articles/PMC5614231/.

14. Colaizy TT, Kuforiji T, Sklar RS, Pillers D-AM. PCR methods in clinical investigations of human ureaplasmas: a minireview. Mol Genet Metab. 2003:80(4):389-97.

15. Asif AA, Roy M, Ahmad S. Rare case of Ureaplasma parvum septic arthritis in an immunocompetent patient. BMJ Case Rep. 2020;13(9):e236396.

16. Meygret A, Le Roy C, Renaudin H, Bébéar C, Pereyre S. Tetracycline and fluoroquinolone resistance in clinical Ureaplasma spp. and Mycoplasma hominis isolates in France between 2010 and 2015. J Antimicrob Chemother. 2018;73(10):2696-703.

\section{Publisher's Note}

Springer Nature remains neutral with regard to jurisdictional claims in published maps and institutional affiliations. 